\title{
Effect of High Electric Fields on the Isotropic Phase of a Lyotropic Liquid-Crystalline System
}

\author{
T. J. MENNA, F. E. FILISKO \\ University of Michigan, Materials Science and Engineering, Ann Arbor, Michigan 48109
}

Received 28 August 2003; revised 20 May 2004; accepted 31 May 2004

DOI: 10.1002/polb.20219

Published online in Wiley InterScience (www.interscience.wiley.com).

\begin{abstract}
The effect of high $(>1000 \mathrm{~V} / \mathrm{mm})$ electric fields on solutions of a lyotropic liquid-crystalline polymer, $\operatorname{poly}(n$-hexyl isocyanate) in $\mathrm{p}$-xylene, is presented. The concentrations are adjusted such that the solutions are strictly within the isotropic phase domain region, thus exhibiting no spontaneous liquid crystallinity. The effects of field strength, frequency, and concentration are varied and the morphological changes are noted. The results are analyzed with birefringence measurements via comparison with the optical Kerr effect. (C) 2004 Wiley Periodicals, Inc. J Polym Sci Part B: Polym Phys 42: 4116-4125, 2004
\end{abstract}

Keywords: liquid-crystalline polymers (LCP); morphology; photoreactive effect; Kerr effect; electric field

\section{INTRODUCTION}

$\operatorname{Poly}(n$-hexyl isocyanate), (PHIC), is a polymer known to exhibit lyotropic liquid crystallinity in the appropriate solvent at moderate concentrations. An applied electric field across these ${ }^{1}$ and other ${ }^{2}$ lyotropic solutions has been shown to greatly increase the rheological properties of the solution. Much of the research to date has focused on these electrorheological (ER) enhancements when the material is actually in the nematic liquid-crystalline state $e^{1,3,4}$ at ambient conditions. However, low concentration solutions, those not spontaneously exhibiting the liquid-crystalline state, or isotropic ones, can be shown to exhibit similar electrorheological behavior, albeit at a smaller scale. Tse and Shine ${ }^{5}$ associate the ER behavior of fully nematic solutions of PHIC in p-xylene with the orientation of the mesogens (PHIC molecules)

\footnotetext{
Correspondence to: T. J. Menna (E-mail: tmenna@ engin.umich.edu)

Journal of Polymer Science: Part B: Polymer Physics, Vol. 42, 4116-4125 (2004) () 2004 Wiley Periodicals, Inc.
}

with the electric field. Another explanation proposed in regard to the ER behavior of suspensions of high viscosity side chain liquidcrystalline polymers, dispersed as fine droplets into low viscosity demethylsiloxane liquids, is related to the liquid-crystalline droplets or domains forming a network between the electrodes under the influence of the electric field. ${ }^{3}$ A further explanation was later proposed in a study by Tajiri el. al., ${ }^{6}$ in which they observed distortion of the droplets by the electric field in the direction of the electrodes to the extent that one may bridge the gap between the electrodes or coalesce with other elliptical droplets to bridge the gap. In these studies the fluids were either fully nematic or they were dispersions. However, in our study, the fluids are homogeneous and isotropic and the first explanation would seem more likely to be applicable. However, it is possible that the effect of the electric field is to induce the formation of a nematic phase in the ambient isotropic solution, a socalled paranematic state ${ }^{5}$ and the latter explanations may apply. 


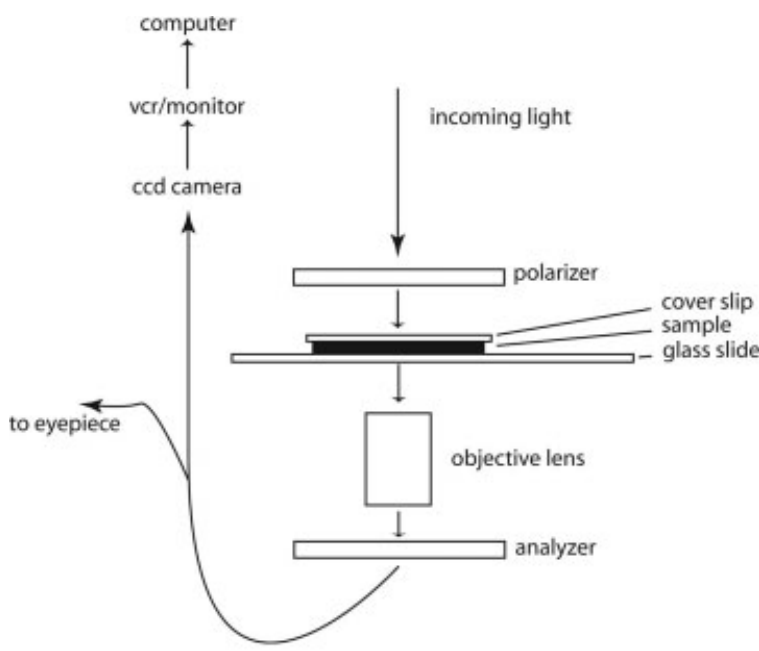

(A)

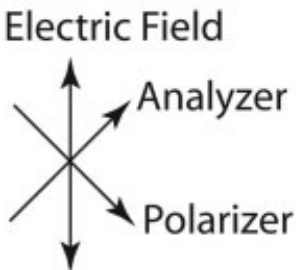

(B)

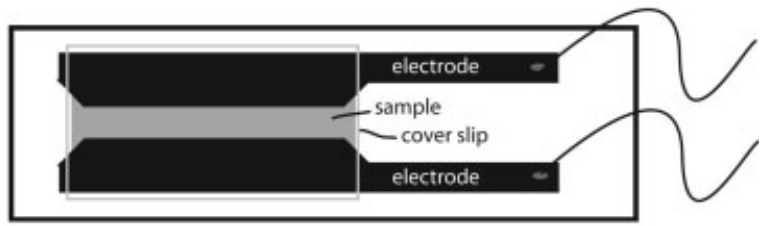

(C)

Figure 1. (a) Electrically induced birefringence instrumentation set-up. (b) Fieldpolarizer-analyzer orientation relative to (c), the electrode used.

Measurements following the change in birefringence of a material with respect to the presence of an externally applied electric field were first noted by $\mathrm{Kerr}^{8}$ in 1875. Many experimentalists (refs. 9-13 example) have since used this observation to describe the effects of an electric field on both the structural and rheological properties of lyotropic and thermotropic liquidcrystalline solutions. Using birefringence, Coles et al. ${ }^{14}$ have shown that dilute PHIC/toluene solutions can be oriented by low strength electric fields and that the electrically induced birefringence values tend to follow the Kerr law, that is, they are proportional to the square of the field magnitude. Other researchers ${ }^{1,2}$ have detected changes in the birefringence patterns because of the alignment of nematic domains of PHIC in p-xylene solutions with alternating current (ac) electric fields.

The work presented here details the effect of an ac electric field on the isotropic phase of the lyotropic liquid-crystalline polymer PHIC in solution with p-xylene. This system is uniquely applicable because it is the only liquid-crystalline polymer that can be dissolved in a nonelectrically conducting solvent to high concentrations $(40-50 \mathrm{wt} \%)$ without forming a highly viscous solution or gel, thus allowing the application of the very high fields $(4000 \mathrm{~V} / \mathrm{mm})$ employed in this study. This article reports the effect of field strength, concentration, and field frequency on the morphological properties of the solutions. Birefringence studies are used in the determination of the relative val- ues of the Kerr constants for these PHIC solutions.

\section{EXPERIMENTAL}

The PHIC was synthesized in our laboratory in a manner similar to that of Shashoua et al. ${ }^{15}$ and Aharoni. ${ }^{16}$ The synthesis method is described elsewhere ${ }^{17}$ and provides, through its methodology, higher yields and better control over the polydispersity. The PHIC used had a weight-average molecular weight of $50,000 \mathrm{~g} / \mathrm{mol}$, as determined from intrinsic viscosity measurements employing the equation of Bur and Fetters, ${ }^{18}$ and a weightaverage degree of polymerization of 394 . Solutions of PHIC and p-xylene (Aldrich) were produced at various weight fractions within the isotropic concentration regime, from zero to the isotropic-nematic phase transition point, determined with cross-polarized light, of $27 \mathrm{wt} \%$.

The solution was placed between parallel electrodes (1.1 mm gap) and observed by the method illustrated in Figure 1. A Trek Model 664 high voltage power supply coupled with a Kikusui Model 455 function generator for frequency applications supplied the electric fields. Light intensity measurements of these solutions were determined under cross-polarized light with a Hamamatsu HC124-02 wideband photomultiplier tube (PMT) with a high-speed amplifier attached to the side port of a Nikon Diaphot 200 inverted stage microscope. The polarizer and analyzer 

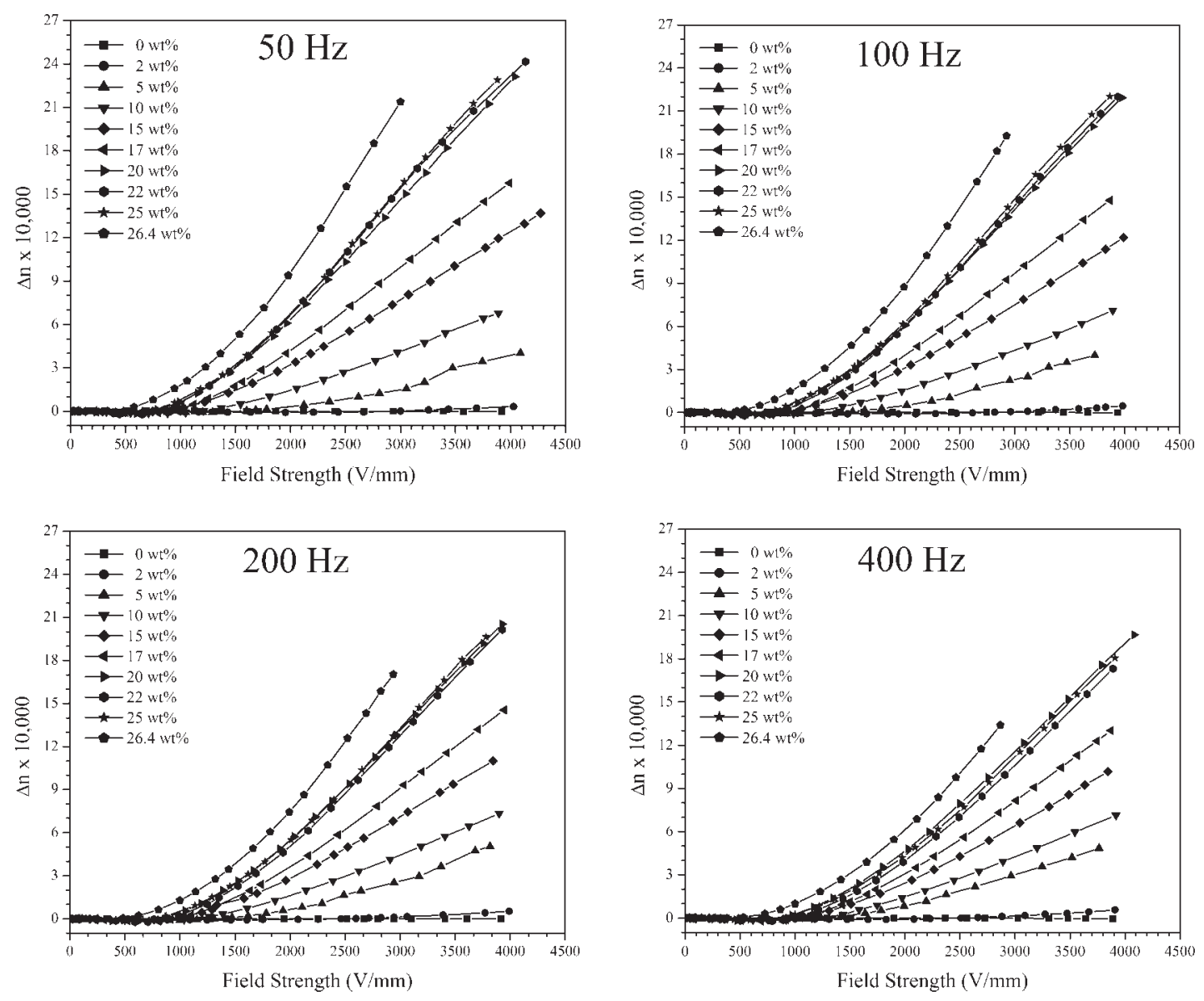

Figure 2. EIB values as a function of field strengths at various frequencies.

were oriented $45^{\circ}$ to the direction of the electric field. Individual sample runs were conducted at various ac field strengths at frequencies of 50 , 100,200 , or $400 \mathrm{~Hz}$. The magnification used was $50 \times$. All testing was done at $23^{\circ} \mathrm{C}$.

The PMT produced values of the light intensity in the form of direct current (dc) volts, read by a Greenlee ${ }^{\oplus}$ DM-200 digital multimeter. Noncrosspolarized $\left(0^{\circ}\right.$ alignment) light measurements, $I_{\mathrm{o}}$, were made at zero applied field to determine the light intensity of the sample itself for comparison to changes, if any, with the field. Field-induced birefringence values, $\Delta n$, were calculated with the derived equation ${ }^{19}$

$$
\Delta n=\frac{\lambda}{\pi d} \sin ^{-1}\left(\sqrt{\frac{I}{I_{0}}}\right)
$$

where $I$ represents the cross-polarized $\left(90^{\circ}\right)$ light intensities, $d$ the sample thickness, $39.5 \mu \mathrm{m}$, and $\lambda$ the characteristic wavelength of the incoming light. A green interference filter was inserted before the polarizer to set the value of $\lambda$ to $546 \mathrm{~nm}$. To avoid the possibility of stray light interference, all measurements were conducted in a darkened room.

\section{RESULTS}

\section{EIB Measurements}

Figure 2 plots the electrically induced birefringence (EIB) as a function of applied electric field strength at various frequencies. Significant increases in EIB occur for concentrations greater than about 5 wt $\%$ and fields greater than approximately $800 \mathrm{~V} / \mathrm{mm}$, and increase steadily with increases in the electric field. At a constant electric field, an increase in solution concentration significantly increases the measured EIB at all frequencies studied. However, between 20 and 25 wt $\%$ the change in concentration has little effect 


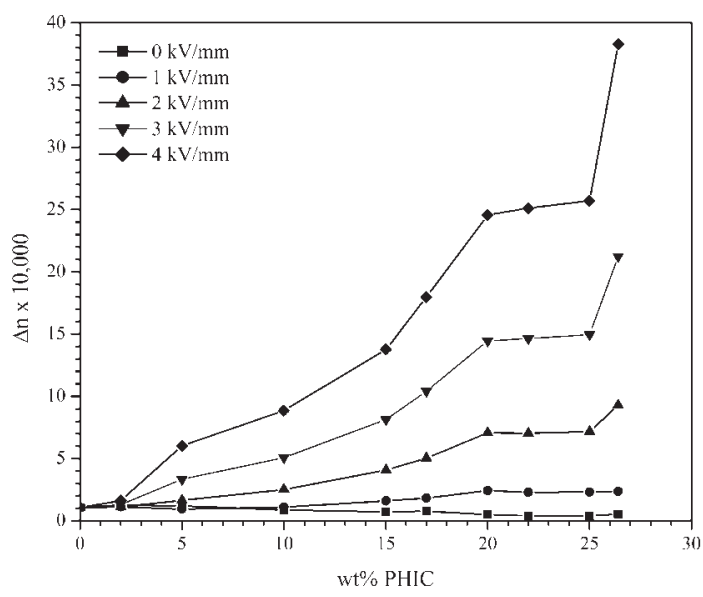

(A)

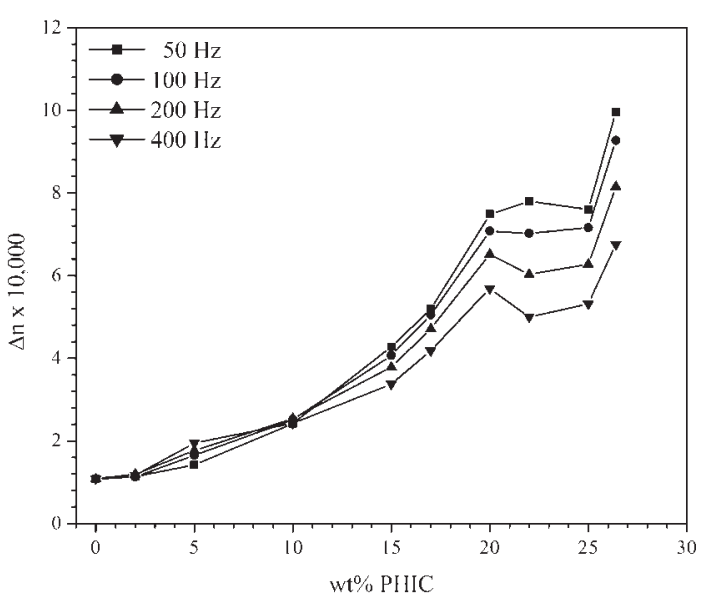

(B)

Figure 3. (a) EIB as a function of concentration at various applied electric fields at $100 \mathrm{~Hz}$. (b) EIB as a function of concentration at a various frequencies at $2000 \mathrm{~V} / \mathrm{mm}$.

upon the EIB, showing only a slight increase with increasing concentration. Above $25 \mathrm{wt} \%$, the concentration effect is again prevalent and the EIB increases. At a constant field strength, the frequency has the effect of reducing the maximum attainable EIB at a particular concentration, more so as the frequency is increased.

Figure 3(a) shows the effect of the electric field on the EIB of a particular concentration. The figure shows that, at a frequency of $100 \mathrm{~Hz}$, the EIB increases monotonically with both the electric field and concentration, except possibly in the region between 20 and 25 wt \%. Increasing concentration increases the EIB roughly exponentially up to 20 wt $\%$, plateaus between 20 and 25 wt $\%$, and then increases again sharply above 25 wt \%. Similar behaviors occur at other frequencies, as illustrated in Figure 3(b) for a constant field strength of $2000 \mathrm{~V} / \mathrm{mm}$. Increasing frequency will decrease the measured EIB. The plateau region, that is, between 20 and 25 wt \% PHIC, although nearly independent of concentration is a strong function of both electric field and frequency, increasing with an increasing electric field and decreasing with an increasing frequency.

With the equation ${ }^{20}$

$$
\Delta n=\frac{1}{2}\left(3 \cos ^{2} \theta-1\right)
$$

the relationship between the EIB and the average angular orientation $\theta$ (in radians) of the PHIC molecules with respect to the field direction can be determined. This relationship is shown in Fig- ure 4 at $100 \mathrm{~Hz}$, with other frequencies showing similar behaviors. The figure indicates a decrease in the deviation from orientation with the electric field direction as the field strength increases, that is, the molecules align more preferentially with the field. When no field is present, the net orientation of the molecules is roughly $55^{\circ}$ from the field direction, showing no concentration dependence. The figure also indicates that a substantially large field is necessary to obtain near perfect alignment with the electric field. As shown in Figure 5, this perfect field strength, $E_{\mathrm{p}}$, decreases with increasing solution concentration in an approximately exponential manner. At a given concentration, increasing the frequency tends to increase the $E_{\mathrm{p}}$.

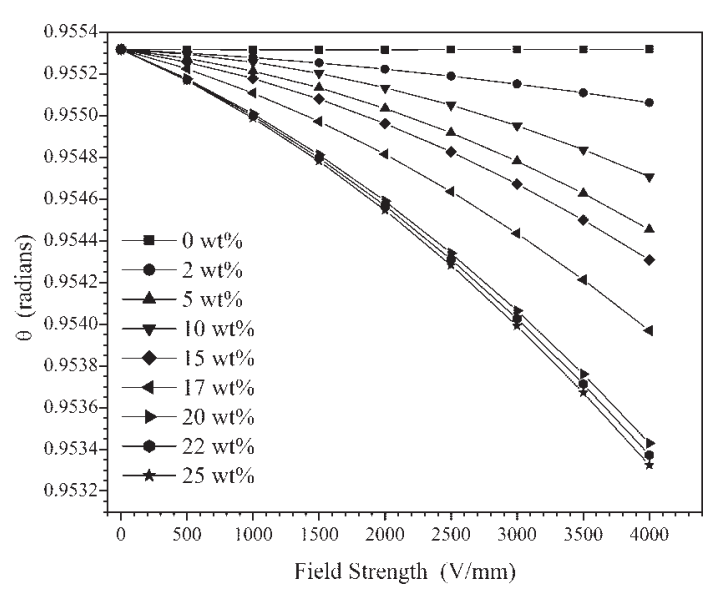

Figure 4. Average orientation angle of the PHIC molecule as a function of electric field strength at $100 \mathrm{~Hz}$. 


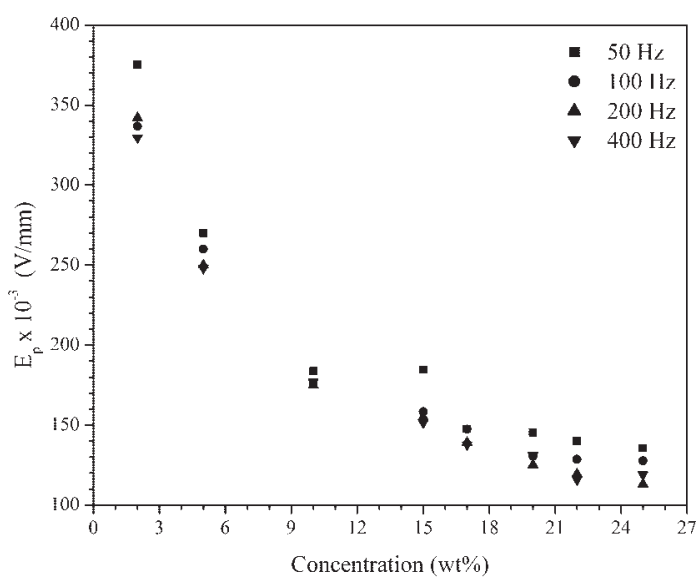

Figure 5. $E_{\mathrm{p}}$ variation with concentration at multiple field frequencies.

Figure 6 illustrates the relationship between the applied field and the EIB at a constant frequency (exhibited in Figure 2) with the use of a power law fit. The figure illustrates the relationship at $400 \mathrm{~Hz}$, other frequencies exhibiting similar behaviors. Straight-line power law fits were used to determine the power law exponents. Because no change in EIB occurs until approximately $800 \mathrm{~V} / \mathrm{mm}$, only field strengths larger than this are represented in the figure. Table 1 lists the power law exponent values at all relevant frequency-concentration combinations. The PHIC solutions all exhibit a positive slope with the EIB increasing with the electric field strength. Solution concentrations less than $10 \mathrm{wt} \%$ do not show a satisfactory power law dependence of EIB on the magnitude of the applied electric field. Above $10 \mathrm{wt} \%$, the solutions exhibit a satisfactory power law fit and the exponents increase steadily with an increase in concentration. Concentrations between 20 and $26 \mathrm{wt} \%$ show an approximate fieldsquared dependence. In this region, the EIB is also independent of concentration. For all data other than the $26.4 \mathrm{wt} \%$, an increase in applied frequency will increase the power law exponent.

Figure 7(a) depicts the data from Table 1 at $2000 \mathrm{~V} / \mathrm{mm}$; a modest value of the field at which the power law relationships fit best. As seen in the figure, the power law exponents vary somewhat logarithmically with the increase in concentration. Figure 7(b) shows the dependence of the power law exponents with frequency at various concentrations. The exponents remain relatively constant with increasing frequency within the range of 50 to $400 \mathrm{~Hz}$. However, the 20 and $25 \mathrm{wt}$ $\%$ concentrations seem to indicate a slight in- crease in the power law exponent with increasing frequency.

\section{Kerr Effect}

Although not strictly followed for low concentrations $(0-20 \mathrm{wt} \%)$ and low fields, for high concentrations (20-26 wt \%) and high fields, the susceptibility of the PHIC to electric field orientation, as measured by the optical Kerr constant, $B$, for our solutions can be determined with the Kerr law:

$$
\Delta n=B \lambda E^{2}
$$

where $\lambda$ is the wavelength of the light and $E$ is the electric field strength. The Kerr constant values were calculated and are shown in Table 2 for each concentration-frequency combination for which the power law exponent was greater than or equal to 1.90 , an exponent value suitably close to the Kerr relationship requirement of 2.00. From Table 1 , only concentrations of and above $20 \mathrm{wt} \%$ satisfy this criterion.

As shown in Table 2 and Figure 8(a), an increase in concentration increases the Kerr constant, regardless of applied frequency. All frequencies show a relatively constant value of the Kerr constant between 20 and 25 wt \%, followed by a sharp increase in magnitude above 25 wt $\%$. However, at a constant concentration, increasing the applied frequency decreases the Kerr constant, as illustrated in Figure 8(b). A point of note is that all Kerr constant values within the 20 to 25 wt \% region can be considered relatively equiv-

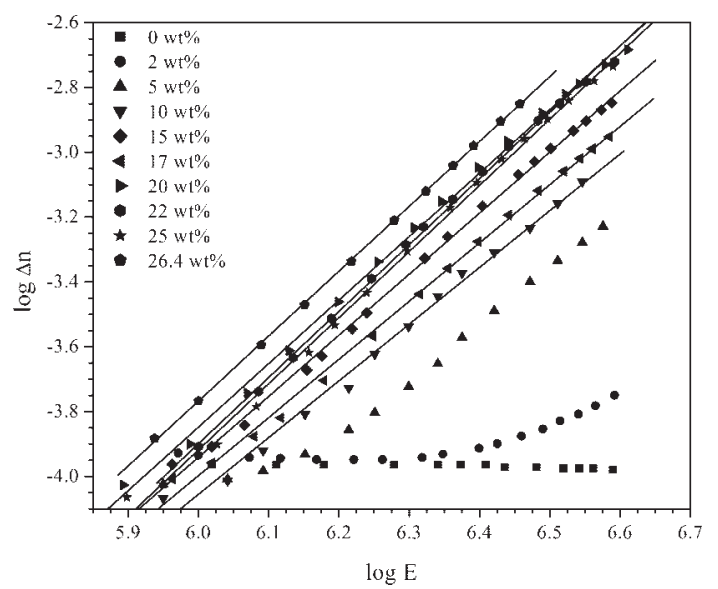

Figure 6. Power law fit analysis at various concentrations at $400 \mathrm{~Hz}$. 
Table 1. Power Law Exponents for Representing the $\Delta n$ Dependency at Various Frequencies

\begin{tabular}{lccrr}
\hline $\begin{array}{c}\text { Concentration } \\
(\text { wt \%) }\end{array}$ & $50 \mathrm{~Hz}$ & $100 \mathrm{~Hz}$ & $200 \mathrm{~Hz}$ & $400 \mathrm{~Hz}$ \\
\hline 10 & $1.64 \pm 0.05$ & $1.59 \pm 0.06$ & $1.63 \pm 0.06$ & $1.70 \pm 0.07$ \\
15 & $1.75 \pm 0.02$ & $1.73 \pm 0.03$ & $1.79 \pm 0.03$ & $1.80 \pm 0.03$ \\
17 & $1.80 \pm 0.02$ & $1.87 \pm 0.02$ & $1.81 \pm 0.03$ & $1.88 \pm 0.02$ \\
20 & $1.91 \pm 0.04$ & $1.92 \pm 0.03$ & $1.96 \pm 0.02$ & $1.99 \pm 0.03$ \\
22 & $1.99 \pm 0.05$ & $2.04 \pm 0.04$ & $2.08 \pm 0.03$ & $2.08 \pm 0.02$ \\
25 & $1.98 \pm 0.04$ & $2.00 \pm 0.04$ & $2.03 \pm 0.02$ & $2.05 \pm 0.02$ \\
26.4 & $2.04 \pm 0.02$ & $2.05 \pm 0.01$ & $2.02 \pm 0.01$ & $2.00 \pm 0.01$ \\
\hline
\end{tabular}

alent, such that a change in concentration will have little effect upon the Kerr constant value.

Extrapolating the curves of Figure $8(\mathrm{~b})$ to a frequency of zero can give us an idea of the relative Kerr constant for these solutions under dc electric field conditions. Doing so, one obtains values of $2.86,2.96,3.07$, and $4.25 \times 10^{-10} \mathrm{~m} / \mathrm{V}^{2}$ for the $20,22,25$, and 26.4 wt $\%$ samples, respectively.

\section{EIB at Low Electric Field Strengths}

As previously noted, at a constant concentration, no significant EIB responses are observed for fields less than or approximately equal to 800 $\mathrm{V} / \mathrm{mm}$. However, as shown in Figure 9, the solutions actually exhibit a variety of behaviors below $800 \mathrm{~V} / \mathrm{mm}$. The figure focuses on the data at 100 and $400 \mathrm{~Hz}$, below a field strength of $1000 \mathrm{~V} / \mathrm{mm}$, at concentrations of 10 or more wt \%. For all concentrations shown, there is an initial decrease in EIB at low field strengths, yielding a negative birefringence. At a particular field strength, the magnitude of which varies with concentration, the curves begin to increase and exhibit a positive birefringence. This critical field strength decreases with increasing concentration and ranges from approximately $900 \mathrm{~V} / \mathrm{mm}$ for the $10 \mathrm{wt} \%$ solution to $300 \mathrm{~V} / \mathrm{mm}$ for the $26.4 \mathrm{wt} \%$ solution. Beyond $800 \mathrm{~V} / \mathrm{mm}$, the influence of concentration on the EIB and an increase in the electric field will increase the birefringence, the rate of increase being dependent on the concentration of the solution, as noted for the data in Figure 2.

At higher frequencies, the variation in EIB with concentration becomes much less pronounced, and the solutions respond essentially as equivalents, that is, independent of concentration, as noticed by comparing Figure 9(a) at 100 $\mathrm{Hz}$ with Figure 9(b) at $400 \mathrm{~Hz}$. It is unknown why the 17 wt \% data does not follow this trend, but it is thought to be an experimental anomaly. The

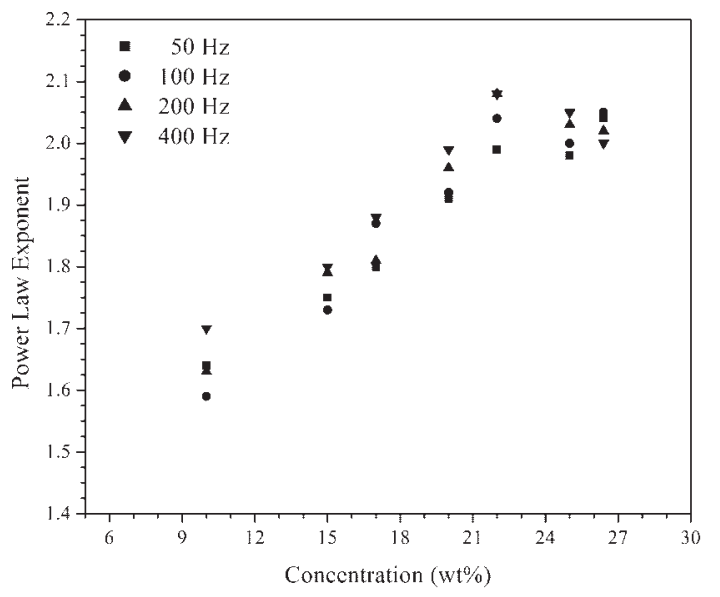

(A)

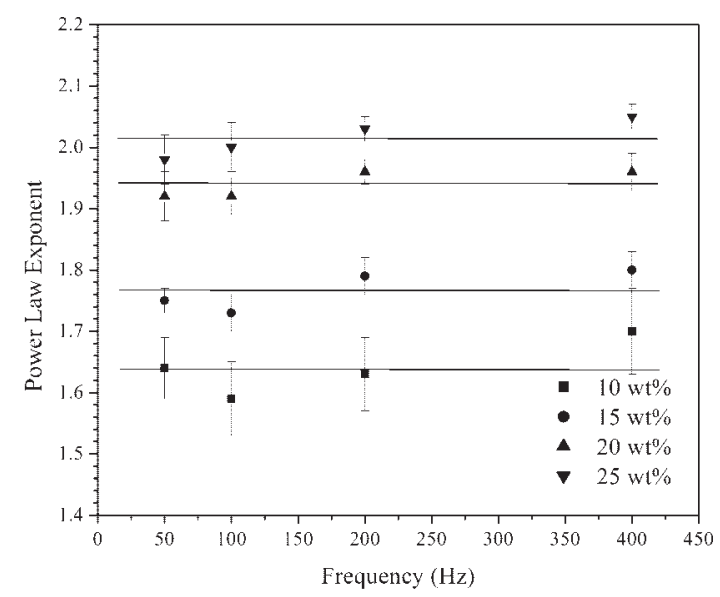

(B)

Figure 7. Dependence of the power law exponent on (a) the concentration at a constant frequency and (b) the frequency at a constant concentration at $2000 \mathrm{~V} / \mathrm{mm}$. 
Table 2. Kerr Constants at Concentrations and Frequencies of the Appropriate Power Law Exponent

\begin{tabular}{ccccr}
\hline & \multicolumn{4}{c}{ Kerr Constant, B $\left(\mathrm{m} / \mathrm{V}^{2}\right)$} \\
\cline { 2 - 5 } $\begin{array}{c}\text { Concentration } \\
\text { (wt \%) }\end{array}$ & $50 \mathrm{~Hz}$ & $100 \mathrm{~Hz}$ & $200 \mathrm{~Hz}$ & $400 \mathrm{~Hz}$ \\
\hline & $2.76 \mathrm{E}-10$ & $2.74 \mathrm{E}-10$ & $2.63 \mathrm{E}-10$ & $2.34 \mathrm{E}-10$ \\
20 & $2.86 \mathrm{E}-10$ & $2.83 \mathrm{E}-10$ & $2.63 \mathrm{E}-10$ & $2.30 \mathrm{E}-10$ \\
25 & $2.98 \mathrm{E}-10$ & $2.89 \mathrm{E}-10$ & $2.73 \mathrm{E}-10$ & $2.36 \mathrm{E}-10$ \\
26.4 & $4.58 \mathrm{E}-10$ & $4.32 \mathrm{E}-10$ & $3.82 \mathrm{E}-10$ & $3.16 \mathrm{E}-10$ \\
\hline
\end{tabular}

curves also exhibit a shift of the critical field strength to higher fields at increased frequencies. The critical field strengths now have a value of near $700 \mathrm{~V} / \mathrm{mm}$, and are relatively constant with concentration; an exception to this is the $26.4 \mathrm{wt}$ $\%$ solution. Although not shown, it should be noted that the $50 \mathrm{~Hz}$ data shows a larger scatter in the EIB values than that of the $100 \mathrm{~Hz}$ data at fields lower than $800 \mathrm{~V} / \mathrm{mm}$, whereas the $200 \mathrm{~Hz}$ samples show data results intermittent between the 100 and $400 \mathrm{~Hz}$ data.

\section{DISCUSSION}

As shown by Filisko et al., ${ }^{21}$ isotropic solutions of PHIC are electrorheologically active down to concentrations of $5 \mathrm{wt} \%$ or less; these are concentrations well below the biphasic transition point of 27 wt \%. Whether domain formations are involved, or if domains are necessarily involved, as suggested by Inoue et al. ${ }^{4}$ and Shine and Yang, ${ }^{22}$ can be suitably answered at this point. Our re- search shows that under a zero field the isotropic state has no domain structure, and upon applying an electric field, there is only a uniformly increasing birefringence with an increasing field (with the exception of the anomalous birefringence behavior at field strengths less than $1000 \mathrm{~V} / \mathrm{mm}$ ), and there is no evidence from this study that domains are induced by the electric field. The possibility of the presence of (induced) domains was investigated primarily by visually observing that these domains may be kinetically different from the electric field oriented isotropically either during or after the application of the electric field.

\section{Effect of the Electric Field}

The behavior at field strengths less than 800 $\mathrm{V} / \mathrm{mm}$ is believed to be related to the phenomenon known as anomalous birefringence. Two possible explanations are worthwhile to investigate. In the first, as suggested by Lauffer ${ }^{23}$ and Mueller and Sakmann, ${ }^{24}$ these anomalies in the response of the EIB at low fields could be explained as a

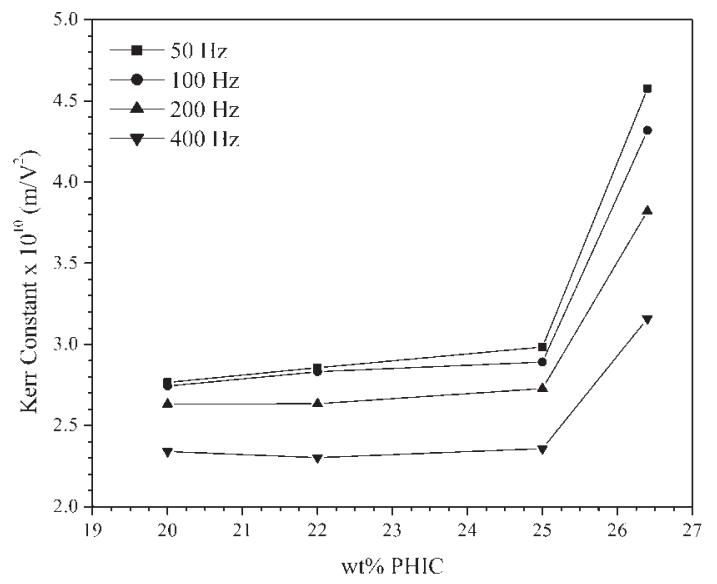

(A)

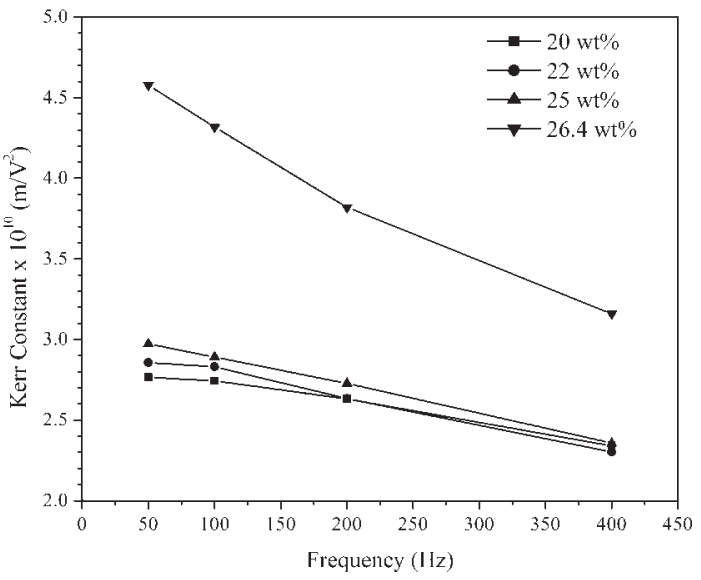

(B)

Figure 8. The effect of (a) concentration at a constant frequency and (b) frequency at a constant concentration depending on the values of the Kerr constant. 


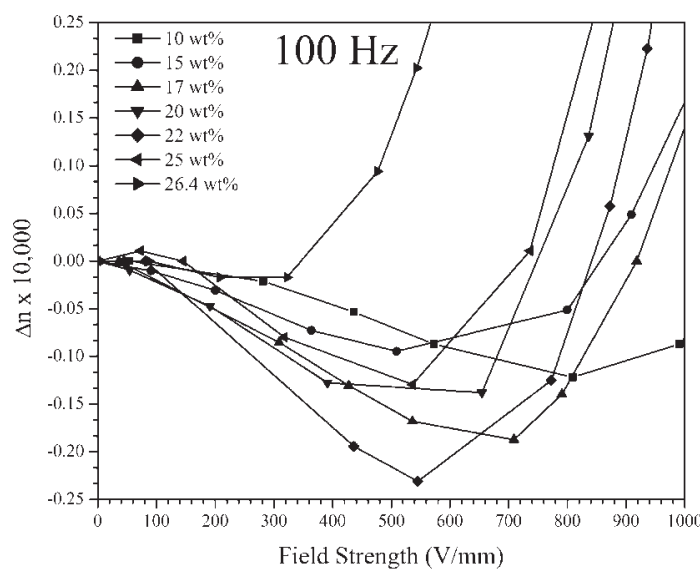

(A)

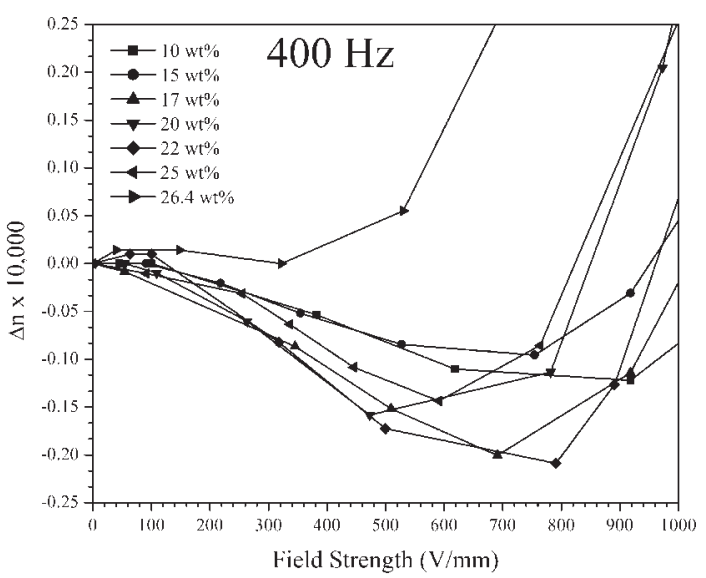

(B)

Figure 9. Anomalous birefringence of PHIC solutions at fields below $800 \mathrm{~V} / \mathrm{mm}$ at (a) 100 and (b) $400 \mathrm{~Hz}$.

superposition of two effects: one being the normal Kerr effect creating the positive birefringence and varying with the second power of the field strength, and the other, similar to the Pockels effect, creating the negative birefringence and varying with the first power of the field strength. The data may be explained by an equation of the type $\Delta n=A E^{2}-B E$, where $A$ and $B$ are constants of proportionality. At low field strengths, the second term will dominate, leading to a decrease in the birefringence. At a critical field strength, the difference in magnitude between the two terms will maximize, and, at increasing fields above this, the first term will overcome that of the second and the birefringence will steadily increase. The value of the critical field strength and rate of increase is noted to decrease and increase, respectively, with an increase in solution concentration or a decrease in the applied field frequency.

The second explanation actually serves as a basis for the first and comes from Hoffman et al. ${ }^{25}$ where, for aqueous solutions containing rod-like micelles, they define the negative birefringence effect as being due to electrostatic interactions of the micelles with their environment, the electrical double layer. At low fields, the double layer creates an induced dipole on the polymer rods in the field direction. This induced dipole will rotate the rod into an alignment perpendicular to the field direction and decrease the EIB. However, with increasing field strength, the double layers of the rods begin to overlap and a network structure develops, allowing for the movement of surface charges, and, hence, reductions in the overall surface charge. Concurrently, the permanent dipole of the PHIC molecules begins to show its field squared dependency and rotates the rod into the direction of the electric field, increasing the EIB. The induced dipole will dominate at the lower fields because of the ease of charge mobility, whereas at higher field strengths the permanent dipole will be dominant. The critical field strength referred to earlier is the point at which the permanent dipole positive EIB overcomes that of the negative EIB-induced dipole of the rods. Theoretically, the electrical double layer reasoning appears to be the dominant explanation, yet because the system we are working with is a solution and the double layer theory needs two separate entities to suffice, the double layer theory is unsatisfactory.

Coles et al. ${ }^{14}$ show no evidence of anomalous birefringence in 90,000 weight-average molecular weight PHIC in toluene solutions. However, the concentrations used in their study were at most $0.5 \mathrm{wt} \%$, that is, $1 / 20$ of our minimum concentration found to exhibit this effect. As noted, the anomalous birefringence does not begin to be prevalent until concentrations near $10 \mathrm{wt} \%$, and, hence, their solutions are not expected to exhibit this phenomenon.

It is well documented (see, for example, ref. 26) that an externally applied electric field will tend to orient molecules in the direction of the applied field, one of the major processes in the phenomenon necessary in the optical Kerr effect. ${ }^{27}$ As the electric field is increased, the average degree of orientation of the PHIC molecules will increase, such that when the field is of sufficient strength, 
all of the molecules will be aligned in the direction of the applied field and the birefringence will approach saturation, leading to an asymptotic value of the EIB. Above this saturation field, Kerr's law is no longer obeyed. ${ }^{28}$ The saturation birefringence at infinitely high fields can be estimated from the small field strength Kerr law: ${ }^{29}$

$$
\Delta n_{\mathrm{sat}}=\frac{15 \Delta n_{\mathrm{o}} k_{\mathrm{B}} T}{\Delta \alpha_{\mathrm{el}} E^{2}}
$$

where $\Delta n_{\mathrm{o}}$ is the measured birefringence, $k_{\mathrm{B}}$ is the Boltzmann constant, $T$ is the absolute temperature, $\Delta \alpha_{\mathrm{el}}$ is the electric anisotropy of the PHIC, and $E$ is the electric field strength. Because of steric interactions and molecular entanglements, higher concentration solutions are expected to reach this asymptotic value at field strengths higher than those of lower concentration solutions. Morgan ${ }^{30}$ reports field strengths of 1.2 to $1.4 \mathrm{kV} / \mathrm{mm}$ necessary to saturate the birefringence of solutions of $80 \mathrm{~S}$ yeast ribosomes in $\mathrm{Mg}$ acetate. We believe that our data represented in Figure 2 is not of sufficient field strength to approach this limit for our solutions, but that saturation should indeed occur at higher field strengths.

\section{Effect of Frequency}

Because the electric field is a function of the frequency, $E=E_{\mathrm{o}} \sin \omega t$, the EIB is expected to be a function of frequency. At sufficiently low frequencies, the molecules are able to follow the field oscillations. However, as the frequency increases, the ability of the molecule to follow the field decreases, and their corresponding electric field response decreases as well. At a sufficiently high frequency, the molecules become unable to follow the field. At frequencies above this critical frequency, no change in EIB is witnessed with an increase in frequency. ${ }^{31,32}$ This behavior is noted in regard to Figures 3, 8(b), and 9, where an increase in frequency will decrease the EIB and Kerr constant values at constant field strengths. The same effect is exhibited by all samples used in our work, regardless of concentration. These effects related to an increase in frequency are found to be consistent with that of other researchers for systems of encapsulated liquid crystals, ${ }^{33}$ dilute rigid-body suspensions, ${ }^{34}$ and rigid chain polymer solutions. ${ }^{35}$

\section{Kerr Law Behavior}

Perhaps the most significant observation noted in this work is the transition to Kerr law behavior at concentrations above 20 wt \%. These concentrations approach the critical isotropic-nematic transition concentration of $27 \mathrm{wt} \%$, and perhaps this is the key to resolving this behavior. Nematic solutions of thermotropic ${ }^{19}$ liquid crystals have been shown to obey the Kerr law, and the research presented here shows that isotropic PHIC/ $\mathrm{p}$-xylene solutions do not. There is no reason to believe that lyotropic and thermotropic liquidcrystalline solutions would not behave similarly in the nematic state. Within the concentration region just below the isotropic-nematic point, the so-called paranematic state is present and the research presented here shows the development and occurrence of Kerr law behavior. At high isotropic concentrations, the induced ordering within the solution created by the presence of the electric field causes the development of the paranematic structures that in turn respond to the field in a manner similar to that of the fully nematic structures. Evidence of this is given by the increase in the power law exponent (Table 1) with increasing concentration, toward a value of 2.00 .

\section{CONCLUSIONS}

The morphological study presented here suggests a correlation between the molecular orientation and the electrorheological behavior ${ }^{36}$ of the PHIC/ $\mathrm{p}$-xylene solutions at concentrations within the isotropic phase region. Clearly, the isotropic phase of a lyotropic liquid-crystalline polymer does respond to the application of an applied ac electric field and an increase in orientation of the molecules results. This orientation was measured by a change in the birefringence of the solution and found to increase with increasing concentration and electric field strength, and decrease with an increase in the frequency of the applied field. Further research is being conducted to investigate these and other behaviors for these solutions within all concentration regimes.

\section{REFERENCES AND NOTES}

1. Yang, I-K.; Shine, A. D. J Rheol 1992, 36, 10791104. 
2. Tanaka, K.; Akiyama, R.; Takada, K. J Appl Polym Sci 1997, 66, 1079-1084.

3. Inoue, A.; Maniwa, S. J Appl Polym Sci 1996, 59, 797-802.

4. Inoue, A.; Ide, Y.; Maniwa, S.; Yamada, H.; Oda, H. MRS Bull 1998, 23, 43.

5. Tse, K.; Shine, A. D. Macromolecules 2000, 33, 3134-3141.

6. Tajiri, K.; Ohta, K.; Nagaya, T.; Orihara, H.; Ishibashi, Y.; Doi, M.; Inoue, A. J Rheol 1997, 41, 335-341.

7. Martin, D. C. Polymer 2002, 43, 4421-4436.

8. Kerr, J. Philos Mag 1875, 4, 337-348.

9. Conradi, M.; Kityk, A. V.; Skarabot, M.; Blink, R.; Musevic, I. Liq Cryst 1999, 26, 1179-1184.

10. O'Konski, C. T.; Haltner, A. J. J Am Chem Soc 1956, 78, 3604-3610.

11. Wegener, W. A. J Chem Phys 1986, 84, 5989-6004.

12. Thurston, G. B.; Bowling, D. I. J Colloid Interface Sci 1969, 30, 34-45.

13. Gatzonis, Y.; Siddiquee, S. K.; van Egmond, J. W. Macromolecules 1997, 30, 7253-7262.

14. Coles, H. J.; Gupta, A. K.; Marchal, E. Macromolecules 1977, 10, 182-187.

15. Shashoua, V. E.; Sweeny, W.; Tietz, R. J Am Chem Soc 1960, 82, 866 .

16. Aharoni, S. M. Macromolecules 1979, 12, 94-103.

17. Hopkins, A. R. Master's Thesis, University of Michigan, 1993.

18. Bur, A. J.; Fetters, L. J Macromolecules 1973, 6, 874.

19. Sinha, A.; Prasada Rao, T. A.; Murthy, V. R. K.; Dabrowski, R. Liq Cryst 2000, 27, 191-194.

20. Fuller, G. G. Optical Rheometry of Complex Fluids; Oxford University Press: New York, 1995; p 113.
21. Filisko, F. E.; Henley, S.; Quist, G. J. Intell Mater Syst Struct 1998, 9, 616-620.

22. Shine, A. D.; Yang, I-K. J Rheol 1992, 35, 1375.

23. Lauffer, M. J Am Chem Soc 1939, 61, 2412-2416.

24. Mueller, H., Sakmann, B. W. Phys Rev 1939, 56, 615-616.

25. Hoffman, H., Krämer, U.; Thurn, H. J Phys Chem 1990, 94, 2027-2033.

26. Blinov, L. M.; Chigrinov, V. G. Electrooptic Effects in Liquid Crystal Materials. Springer-Verlag: New York, 1994.

27. Hanus, J Phys Rev 1969, 178, 420-428.

28. Aroney, M. J. Angew Chem 1977, 16, 663-734.

29. Kramer, H.; Graf, C.; Hagenbüchle, M.; Johner, C.; Martin, C.; Schwind, P.; Weber, R. J Phys II 1994, 4, 1061-1074.

30. Morgan R. S. Biophys J 1963, 3, 253-259.

31. Riande, E.; Saiz, E. Dipole Moments and Birefringence of Polymers; Prentice Hall: New Jersey, 1992; pp 193-259.

32. O'Konski, C. T.; Haltner, A. J. J Am Chem Soc 1957, 79, 5634-5649.

33. Sansone, M. J.; Khanarian, G.; Kwiatek, M. S. J Appl Phys 1994, 75, 1715-1721.

34. Wegener, W. A. J Chem Phys 1986, 84, 6005-6012.

35. Tsvetkov, V. N. Rigid-Chain Polymers: Hydrodynamic and Optical Properties in Solution; Consultants Bureau: New York, 1989.

36. Menna, T. J. Response of a Poly(n-hexyl isocyanate)/p-Xylene Solution to Applied High Electric Fields and Shear; presented at the Society of Rheology $74^{\text {th }}$ Annual Meeting, Minneapolis, Minnesota, October 2002. 